\title{
Calculation of Gamma-ray Mass Absorption Coefficients for some Geological Compounds for Energy of 10-150 keV
}

\author{
Samir A. Hamouda ${ }^{* 1}$, Essam al-Talhi ${ }^{\# 2}$, Mohamed B. Abdelmalik ${ }^{\# 3}$ \\ ${ }^{I}$ Associate Professor, Department of Physics, University of Benghazi, Libya \\ drshamouda@yahoo.ie \\ ${ }^{2}$ Ph.D. Scholar, Department of Physics, University of Benghazi, Libya \\ ${ }^{3}$ Associate Professor, Department of Earth Sciences, University of Benghazi, Libya
}

\begin{abstract}
Information about gamma-ray mass absorption coefficients in different geological compounds with different percentage to their rations is vital for accurate gamma-ray spectroscopy analysis. In this paper, gamma-ray absorption coefficients for energy (10-150 keV) were calculated for Corundum $\left(\mathrm{AL}_{2} \mathrm{O}_{3}\right)$, Quartz $\left(\mathrm{SiO}_{2}\right)$, Calcite $\left(\mathrm{CaCO}_{3}\right)$, Hematite $\left(\mathrm{Fe}_{2} \mathrm{O}_{3}\right)$, and Magnetite $\left(\mathrm{Fe}_{3} \mathrm{O}_{4}\right)$ compounds with their \% mixtures.
\end{abstract}

Keywords - Mass Absorption Coefficients; Gamma-Ray Spectroscopy; Geology; Mixtures.

\section{Introduction}

The most important description of the amount of radiation penetration into the material is the Linear Attenuation Coefficient, which is a quantity that depends on the energy of the incident photon and the atomic number of the material. This quantity represents a fraction of the energy lost from the incident photon for every $1 \mathrm{~cm}$ penetrated through the material. The unit of linear absorption coefficient $\mu$ is $\mathrm{cm}^{-1}$. According to Lambert Beer's law [1-6], when a narrow photon beam of singleenergy $h v_{0}$ and a flux density $\mathrm{N}_{0}$ (the number of photons per unit area and time) falls on a homogeneous medium and penetrates it a distance $(x)$, the flux density of the transmitted photon beam from this medium is $\mathrm{N}$ and is given as:

$$
N(\mathrm{X})=N_{0} e^{-\mu x}
$$

This law shows that the transmitted photons decrease exponentially with the thickness of the absorbent material. It can be stated that, when photon energies are greater than electron binding energies, $\mu$ is directly proportional to the physical density of the absorbent material $[5,7]$. Therefore, equation (1) can be rewritten as follows:

$$
N=N_{0} e^{-\left[\frac{\mu}{\rho}\right] \mathrm{x}_{0}}
$$

Where $(\mu / \rho)$ is the mass absorption coefficient $\left(\mathrm{cm}^{2} / \mathrm{g}\right)$. This quantity $(\mu / \rho)$ is related to the probability (or crosssection) that the beam will interact with matter and contribute in some way. Each interaction somehow contributes to the loss of a number of photons from the incident photon ray even though the scattering of the photon does not lead to its absorption from the photon beam. This probability gives a measure of the mass absorption of the photons that pass through the medium and since there are three basic interactions that occur when the photon interacts with the material, and these interactions are the phenomenon of photoelectric absorption (ph), Compton scattering (C) and Rayleigh scattering (R), the mass absorption coefficient It can be written as follows:

$$
\left(\frac{\mu}{\rho}\right)=\left(\frac{\mu}{\rho}\right)_{p h}+\left(\frac{\mu}{\rho}\right)_{C}+\left(\frac{\mu}{\rho}\right)_{R}
$$

\section{Mass Absorption Coefficient of Mixtures}

When the absorbent material is a homogeneous mixture of different types of elements (chemical compound) or a mixture, the mass attenuation coefficient is given as [6]

$$
\mu / \rho=\sum_{j} w_{j} \mu_{j} / \rho_{j}
$$

Where $w_{j}$ represents the weight of the component $j, \mu_{j}$ represents the element's linear attenuation coefficient for element $\mathbf{j}$ and $\rho_{\mathrm{j}}$ is the density of element $\mathrm{j}$.

\section{Calculation of the Mass Absorption Coefficient of some Geological Components}

The mass absorption coefficients for Corundum $\left(\mathrm{AL}_{2} \mathrm{O}_{3}\right)$, Quartz $\left(\mathrm{SiO}_{2}\right)$, Calcite $\left(\mathrm{CaCO}_{3}\right)$, He mat ite $\left(\mathrm{Fe}_{2} \mathrm{O}_{3}\right)$, and Magnetite $\left(\mathrm{Fe}_{3} \mathrm{O}_{4}\right)$ compounds with their \% mixtures were calculated as a function of incident photon energies $(10-150 \mathrm{keV})$. Table 1 shows the mass absorption coefficient of a mixture in terms of the energy of the incident photon.

Figure1 shows the calculated mass absorption coefficients for $\mathrm{AL}_{2} \mathrm{O}_{3}, \mathrm{SiO}_{2}$ and $\mathrm{Fe}_{3} \mathrm{O}_{4}$ as a function of incident photon energies. It can be seen from figure1, that Haematite (density $5.3 \mathrm{gm} . / \mathrm{cm}^{3}$ ) has higher mass absorption coefficients than Corundum (density 4.02 
$\mathrm{gm} . / \mathrm{cm}^{3}$ ) and Quartz (density $2.65 \mathrm{gm} . / \mathrm{cm}^{3}$ ) due to differences in density.

Table1: The calculated mass absorption coefficients for different compounds as a function of incident photon energies

\begin{tabular}{|c|c|c|c|c|c|}
\hline $\begin{array}{l}\text { nergy } \\
(\mathrm{keV})\end{array}$ & $\begin{array}{l}\text { forundum } \\
\mathrm{AL}_{2} \mathrm{O}_{3}\end{array}$ & \begin{tabular}{|l} 
Quartz \\
$\mathrm{SiO}_{2}$ \\
\end{tabular} & $\begin{array}{l}\text { Calcite } \\
\mathrm{CaCO}_{3}\end{array}$ & $\begin{array}{c}\text { Hematite } \\
\mathrm{Fe}_{2} \mathrm{O}_{3}\end{array}$ & $\begin{array}{l}\text { Aagnetite } \\
\mathrm{Fe}_{3} \mathrm{O}_{4}\end{array}$ \\
\hline & \multicolumn{5}{|c|}{ Total mass absorption coefficient $\left(\mathrm{cm}^{2} / \mathrm{g}\right)$} \\
\hline 10 & 39.53 & 25.98 & 61.25 & 272.38 & 407.93 \\
\hline 15 & 11.89 & 7.80 & 19.11 & 87.97 & 131.77 \\
\hline 20 & 05.20 & 3.39 & 8.28 & 40.26 & 60.30 \\
\hline 30 & 01.82 & 1.17 & 2.65 & 12.88 & 19.28 \\
\hline 40 & 00.99 & 0.63 & 1.26 & 5.79 & 8.66 \\
\hline 50 & 00.70 & 0.43 & 0.77 & 3.16 & 4.72 \\
\hline 60 & 00.56 & 0.34 & 0.54 & 1.99 & 2.97 \\
\hline 80 & 00.44 & 0.26 & 0.36 & 1.03 & 1.53 \\
\hline 100 & 00.38 & 0.23 & 0.28 & 0.67 & 1.00 \\
\hline 150 & 00.32 & 0.19 & 0.21 & 0.39 & 0.57 \\
\hline
\end{tabular}

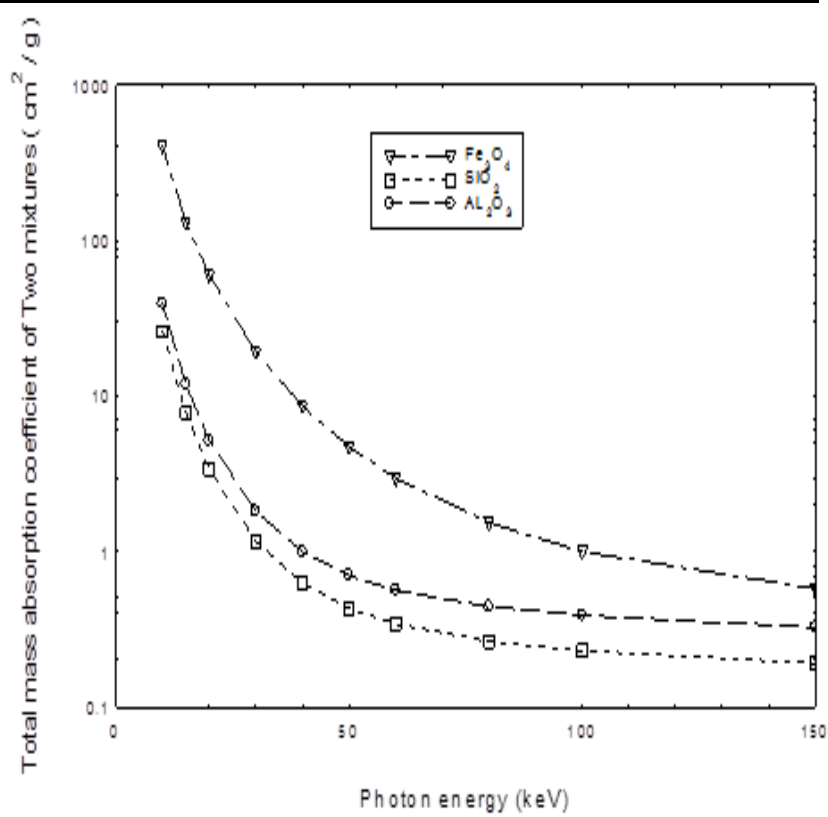

Fig.1: shows mass absorption coefficients for $\mathrm{AL}_{2} \mathrm{O}_{3}, \mathrm{SiO}_{2}$ and $\mathrm{Fe}_{3} \mathrm{O}_{4}$ in the energy range $(10-150 \mathrm{keV})$.

Table2 shows the calculations of the mass absorption coefficients of two mixtures in fixed proportions in terms of the energy of the incident photon.

Figure 2 shows the mass absorption coefficients of two mixtures with $50 \%$ proportions in the photon energy range $(10-150 \mathrm{keV})$. As shown in figure2 that the two curves for the mass absorption coefficients of two mixtures
(CaCO3 $50 \%$; AL2O3 $50 \%$ ) and (SiO2 $50 \%$; AL2O3 $50 \%)$ are very close to each other due to the fact that $(\mathrm{CaCO} 3$ and $\mathrm{SiO} 2)$ have almost the same density values.

Table2: Calculations of the mass absorption coefficients of two mixtures in terms of the energy of the incident photon

\begin{tabular}{|c||c||c||}
\hline $\begin{array}{c}\text { Energy } \\
(\mathbf{k e V})\end{array}$ & $\begin{array}{c}\mathrm{CaCO}_{3} \mathbf{5 0} \% \\
\mathrm{AL}_{2} \mathbf{O}_{3} \mathbf{5 0} \%\end{array}$ & $\begin{array}{c}\mathbf{S i O}_{2} \mathbf{5 0} \% \\
\mathrm{AL}_{2} \mathbf{O}_{3} \mathbf{5 0} \%\end{array}$ \\
\hline \hline & \multicolumn{2}{|c|}{ Mass absorption coefficients $(\mathrm{cm} 2 / \mathrm{g})$} \\
\hline \hline 10 & 50.39 & 32.75 \\
\hline \hline 15 & 15.50 & 9.85 \\
\hline \hline 20 & 6.74 & 4.29 \\
\hline \hline 30 & 2.23 & 1.49 \\
\hline \hline 40 & 1.13 & 0.81 \\
\hline \hline 50 & 0.73 & 0.56 \\
\hline \hline 60 & 0.55 & 0.45 \\
\hline \hline 80 & 0.40 & 0.35 \\
\hline \hline 100 & 0.33 & 0.30 \\
\hline \hline 150 & 0.27 & 0.25 \\
\hline
\end{tabular}

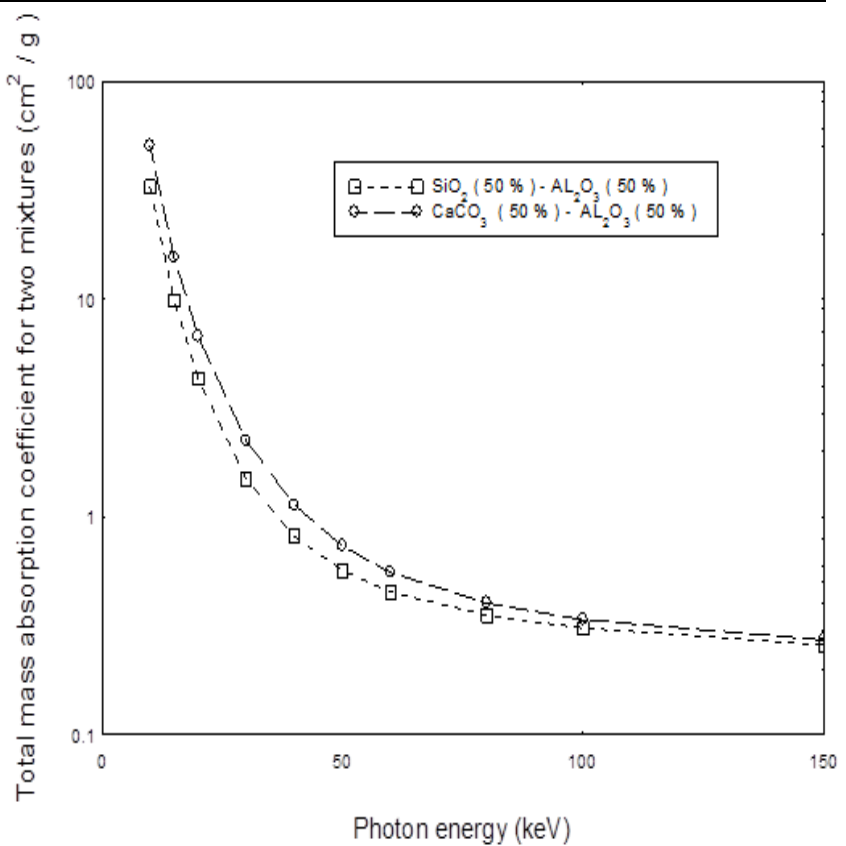

Fig.2: The mass absorption coefficient of two mixtures in the photon energy range $(10-150 \mathrm{keV})$

Table 3 shows the mass absorption coefficient of three compounds with different ratios in terms of the energy of the incident photon.

Figure 3 shows the projection of the calculated mass absorption coefficients of three compounds (SiO2 $20 \%$, CaCO3 $60 \%$ and AL2O3 $20 \%$ ) as a function of incident 
photon energies. This curve shows higher values of mass absorption coefficients for the mixture which made up of light and relatively higher atomic numbers.

Table3: Calculated mass absorption coefficient of three compounds with different ratios as a function of incident photon energies

\begin{tabular}{|c||c|}
\hline $\begin{array}{c}\text { Energy } \\
(\mathrm{keV})\end{array}$ & $\begin{array}{c}\mathrm{SiO}_{2} 20 \% \\
\mathrm{CaCO}_{3} 60 \% \\
\mathrm{AL}_{2} \mathrm{O}_{3} 20 \%\end{array}$ \\
\hline \hline & $\begin{array}{c}\text { Mass absorption coefficients of three } \\
\text { mixtures }(\mathrm{cm} 2 / \mathrm{g})\end{array}$ \\
\hline \hline 10 & 49.85748552 \\
\hline \hline 15 & $\mathbf{1 5 . 4 1 0 1 5 4 5 4}$ \\
\hline \hline 20 & $\mathbf{6 . 6 8 9 5 0 5 3 1 1}$ \\
\hline \hline 30 & $\mathbf{2 . 1 9 2 1 7 8 9 0 1}$ \\
\hline \hline 40 & $\mathbf{1 . 0 8 5 7 2 4 7 0 7}$ \\
\hline \hline 50 & $\mathbf{0 . 6 8 9 5 7 6 3 6 3}$ \\
\hline \hline 60 & $\mathbf{0 . 5 1 0 0 4 2 3 1 3}$ \\
\hline \hline 80 & $\mathbf{0 . 3 5 8 8 2 7 5 6 3}$ \\
\hline \hline 100 & $\mathbf{0 . 2 9 6 4 9 0 3 4 7}$ \\
\hline \hline 150 & $\mathbf{0 . 2 3 4 7 6 6 0 9 2}$ \\
\hline
\end{tabular}

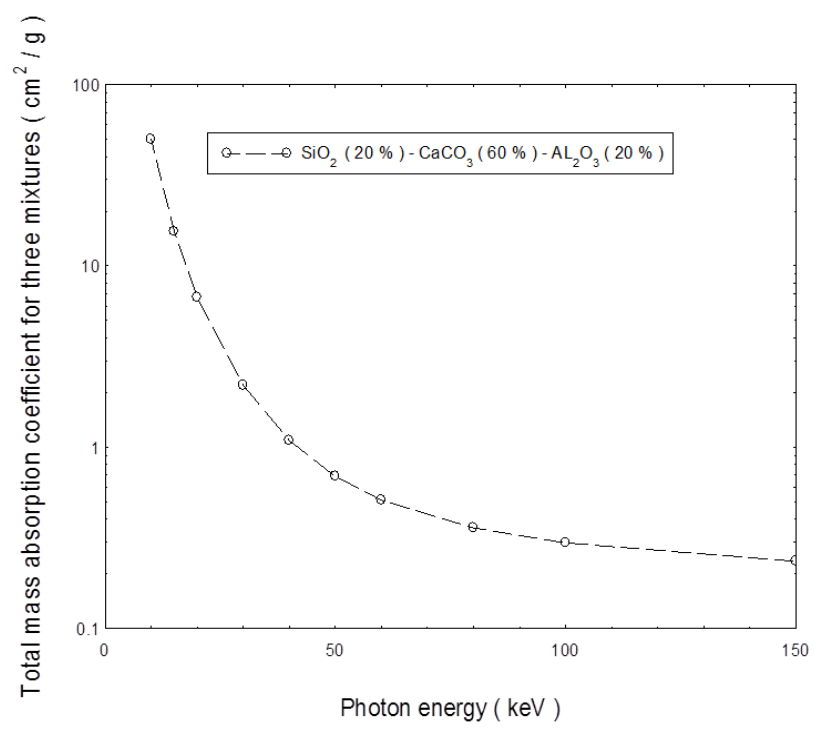

Fig.3: shows the curve of mass absorption coefficient of three compounds with different proportions as a function of incident photon energies (10-150 keV).

\section{Conclusion}

In x-ray and gamma- ray spectroscopy analysis, it is always required appropriate correction for data matrix of geological materials. However, there are many corrections to be taken into account. Such corrections depend on the type of analysis required. One such important correction is mass absorption coefficients. Since the mass absorption coefficients for materials cannot be measured experimentally due to the fact that there are many different scattering processes involved. Therefore, seeking a theoretical method for calculation of mass absorption coefficients of materials appears to be the only method that is available. This paper deals with estimation of mass absorption coefficients for some geological compounds and their mixtures for incident photon energies (10 -150 keV). As seen from the above figures, the mass absorption coefficients curves show exponential decrease as a function of increasing photon energy. This last point has many theoretical and practical applications in different research desplines.

\section{Reference}

[1] W. Heitler, (1955), "Quantum Theory of Radiation", Sec. 26, Oxford

[2] R. D. Evans, (1955), "The atomic nucleus", McGraw Hill Book Company, Inc., New York.

[3] C. M. Davisson and R. D. Evans, (1952), "Gamma-ray absorption coefficient". Rev. Mod. Phys, V24,79-103.

[4] Harvey Hall, (1936), "The theory of photoelectric absorption for xrays and Gamma-rays", Reviews of modern physics, Vol 8, pp358377.

[5] J. Hubbel , (1982), "Photon mass Attenuation and EnergyAbsorption Coefficients from $1 \mathrm{keV}$ to $20 \mathrm{keV}$ ", Int. J. appl. radiat. isot, .vol 33,1269-1290.

[6] National Bureau of Standarads,(1969), "Photon Cross Sections, Attenuation Coefficient, and Energy Absorption Coefficients From $10 \mathrm{keV}$ to $100 \mathrm{GeV}$ ', U.S. Department of Commerce. 